\title{
High-dimensional association detection in large scale genomic data
}

\author{
Hillary Koch \\ Dept. of Statistics, Pennsylvania State University \\ Cheryl A. Keller
}

Dept. of Biochemistry and Molecular Biology, Pennsylvania State University

Belinda Giardine

Dept. of Biochemistry and Molecular Biology, Pennsylvania State University

Guanjue Xiang

Center for Computational Biology and Bioinformatics, Pennsylvania State University

Feipeng Zhang

School of Economics and Finance, Xi'an Jiaotong University

Yicheng Wang

School of Mathematics and Statistics, Lanzhou University

Ross C. Hardison*

Dept. of Biochemistry and Molecular Biology, Pennsylvania State University

Qunhua $\mathrm{Li}^{\dagger}$

Dept. of Statistics, Pennsylvania State University

November 18, 2020

\begin{abstract}
Joint analyses of genomic datasets obtained in multiple different conditions are essential for understanding the biological mechanism that drives tissue-specificity and cell differentiation, but they still remain computationally challenging. To address this we introduce CLIMB (Composite LIkelihood eMpirical Bayes), a statistical methodology that learns patterns of condition-specificity present in genomic data. CLIMB provides a generic framework facilitating a host of analyses, such as clustering genomic features sharing similar condition-specific patterns and identifying which of these features are involved in cell fate commitment. Our approach improves upon existing methods by boosting statistical power to identify meaningful
\end{abstract}

*email: rch8@psu.edu

${ }^{\dagger}$ email: qunhua.li@psu.edu 
signals while retaining interpretability and computational tractability. We illustrate CLIMB's value on two sets of hematopoietic data: one studying CTCF ChIP-seq measured in 17 different cell populations, and another examining RNA-seq measured across constituent cell populations in three committed lineages. These analyses demonstrate that CLIMB captures biologically relevant clusters in the data and improves upon commonly-used pairwise comparisons and unsupervised clusterings typical of genomic analyses.

Uncovering changes across multiple biological conditions is a lasting theme in large-scale genomic data analyses across many types of studies. Examples include the analysis of tissue-specificity of gene expression patterns ${ }^{1,2}$, differential protein binding across cell types ${ }^{3,4,5}$, or causal single nucleotide polymorphisms (SNPs) ${ }^{6,7,8,9}$ and pleiotropic genetic variants ${ }^{10}$ across many genome-wide association (GWA) studies. We are specifically motivated by two contexts:

Motivating context 1 Classification by association patterns: if a set of subjects has been observed in many conditions, one may seek to assign subjects to classes based on the patterns of association they exhibit across biological conditions. For example, when studying plasticity of gene expression across multiple human tissues, joint analysis of these data might ask which sets of genes are collectively up-regulated together in some tissues, but down-regulated in others.

Motivating context 2 Testing for consistent findings across many experiments: one may desire to determine which signals are consistent across studies. For example, if one has several GWA studies examining the same phenotype, one may ask which variants have a consistent association with either the presence or repression of said phenotype.

Both motivating contexts concern determining observations that have either null or significant associations across a collection of conditions. One standard approach to jointly analyzing a collection of conditions applies general clustering algorithms such as K-means or hierarchical clustering. Though these techniques can group signal profiles with similar association patterns together, their results do not directly provide information on condition specificity, such as which signals are consistent or differential across conditions. Somewhat similarly, time series-inspired methods such as the short time-series expression miner ${ }^{11}$ may be applied to genomic data collected at multiple time points. However, this approach assumes a temporal relationship across conditions and groups observations according to changes relative to a temporal baseline. This temporal assumption may not be applicable for studying genetic pleiotropy or plasticity in gene regulation, and again cannot be used to identify patterns of condition specificity. Alternatively, one may identify observations significantly associated with each condition separately, and use these individual outcomes to determine which relationships are significantly shared or differential across conditions. This technique, which is commonly used in expression quantitative trait locus (eQTL) analyses ${ }^{1}$, does not leverage any information-sharing among conditions, and is thus underpowered to identify shared or differential associations ${ }^{12,13}$. Urbut et al. ${ }^{14}$ improved upon single-condition analyses with a statistical model for joint eQTL analysis. This approach shows increased power; however, it makes some restrictive modeling assumptions that are not always appropriate, especially when seeking consistent signals across conditions, as we will illustrate later. Pairwise analyses, commonly employed for differential expression analysis, also improve upon analyses of individual conditions, but still do not offer the power of a joint analysis when more than 2 conditions are present. 
Moreover, when more than 2 conditions are examined, it is unclear how to properly aggregate findings from a series of pairwise comparisons.

To provide interpretable joint analysis of multiple conditions, several others have introduced "association vectors" to describe an observation's specific pattern of association across conditions; these approaches leverage mixture models to cluster observations into groups with different association vectors. For example, Andreassen et al. ${ }^{10}$ apply association vectors to the study of pairs of GWA studies. In this two-condition setting, they assume the presence of 4 association vectors $\{(0,0),(0,1),(1,0),(1,1)\}$, where a SNP described by the $(0,0)$ assocation vector is null in both studies, a SNP from $(1,1)$ is non-null in both studies, and a SNP from $(0,1)$ or $(1,0)$ is null in one of the studies, but non-null in the other. Some ${ }^{15,16}$ similarly use association vectors to find reproducible observations across replicated experiments, while others ${ }^{17,18}$ leverage them to determine which SNPs are eQTLs across various tissues.

These association vectors can be appreciated as an alternative to binarization or ternarization of genomic signals, since they assign binary or ternary labels to the data. A label directly reflects the pattern of condition specificity of the observations in its associated cluster. Further, as a mixture modeling approach, these labels naturally allow for heterogeneity in signals, resulting in greater model flexibility.

Yet, a remaining challenge is that models that leverage these association vectors suffer from computational intractability for even a modest number of conditions ${ }^{15,17}$. To understand this issue, consider $D$ conditions: Let $\mathcal{H}=\left\{H=\left(h_{[1]}, \ldots, h_{[D]}\right): h_{[i]} \in\{-1,0,1\}\right\}$ be the set of all $3^{D}$ possible configurations of association vector $H$, such that an observation described by an association vector with $h_{[i]}=1\left(h_{[i]}=-1\right)$ has a positive (negative) association in condition $i$. It is clear that this model formulation becomes computationally prohibitive even for single-digit $D$ because the total number of possible association vectors grows exponentially with $D$, even resulting in the number of model parameters exceeding the number of observations. In response to this, several restrictive assumptions are imposed. For example, Amar et al. ${ }^{16}$ partially alleviate computational burden by assuming all associations must be positive, and partially estimate the latent associations with a heuristic approach. This heuristic reduces statistical power to test for consistent findings and cannot provide a single unified clustering of observations since it is not a true joint analysis. Moreover, this approach does not distinguish an observation that is significant in opposite directions in two conditions from an observation that exhibits consistent direction of association across conditions. Alternatively, Urbut et al. ${ }^{14}$ make computational gains by assuming all observations come from a uni-modal distribution centered over zero, but this restriction often does not hold in practice.

We present a methodology we refer to as CLIMB (Composite LIkelihood eMpirical Bayes) that allows us to tractably estimate which latent association vectors are likely to be present in the data. Our method is motivated by the observation that the true number of latent classes, each described by a different association vector, cannot be greater than the sample size. Thus, in higher dimensions, the number of true classes is very small relative to $3^{D}$, and many candidate classes have no members. By identifying these classes through a computationally efficient pairwise composite likelihood (CL) model and rigorously filtering out unsupported latent classes, we elucidate sparsity in class membership. In doing so, the aforementioned computational intractability issue falls away, and a joint Bayesian analysis, informed by the initial CL modeling, can be performed in a computationally tractable way. Using ChIP-seq and RNA-seq data collected from hematopoietic 
cell lineages, we demonstrate that CLIMB compares favorably against existing alternatives based on improved statistical power, precision, and model interpretability for investigating cell type-specific protein binding and lineage-specific gene expression patterns.

\section{Results}

\section{Overview of CLIMB}

We model the multi-conditional data using a constrained mixture model that encodes conditionspecificity through latent association labels -1, 0, and 1 (Fig. 1a). The parameter constraints in the model enforce some general patterns commonly observed under condition-specificity: (1) observations that are associated with a condition (i.e., association label \pm 1 ) have a stronger average signal than those that are not (i.e., association label 0), and (2) observations that are associated with multiple conditions correlate with one another within a given cluster. Specifically, we assume the data are summarized as some score, and transformed to a $Z$-score, with larger values corresponding to stronger signals (see Simulations and Case studies for examples of transformation). Then, letting $n$ be the sample size, $D$ be the dimension of the data, and $H=\left(h_{[1]}, \ldots, h_{[D]}\right)$ be a ternary latent association vector, the observed data $\mathrm{x}$ across $D$ conditions follow the normal mixture model

$$
\begin{aligned}
\mathbf{x} \mid H=h_{m} & \sim \phi_{D}^{c}\left(\boldsymbol{\mu}_{m}, \boldsymbol{\Sigma}_{m}, h_{m}\right) \\
H & \sim \operatorname{Mult}\left(\pi_{1}, \ldots, \pi_{M}\right), \quad \sum_{m=1}^{M} \pi_{m}=1
\end{aligned}
$$

where $h_{m}$ is the $m^{\text {th }}$ latent class, $m \in 1, \ldots, M$, and $\phi_{D}^{c}$ is a $D$-dimensional constrained normal distribution. The constrained normal distribution, defined presently, is used to impose association label-driven constraints:

$$
\begin{aligned}
& \phi_{D}^{c}(\mathbf{x} ; \boldsymbol{\mu}, \Sigma, h)=\phi_{D}(\mathbf{x} ; \boldsymbol{\mu}, \Sigma), \text { subject to } \\
& \\
& \operatorname{sgn}\left(\mu_{d}\right)=h_{[d]} \forall d \in\{1, \ldots, D\} \text { and } \\
& \operatorname{sgn}\left(\Sigma_{r t}\right)=h_{[r]} \cdot h_{[t]} \forall r \neq t
\end{aligned}
$$

where $\mu_{d}$ is the $d^{t h}$ element of $\boldsymbol{\mu}$ and $\Sigma_{r t}$ is the $(r, t)^{t h}$ element of $\Sigma$.

Though the possible number of latent classes $M$ explodes combinatorially, many latent classes likely have no members. In order to estimate the actual number of classes, we leverage information about association patterns between pairs of conditions through a pairwise composite likelihood model to eliminate classes that are unlikely to be present in the data, making the final model computationally tractable. This filtering works as depicted through a toy example in Fig. 1b, and is briefly described in four major steps:

1. Pairwise fitting. Fit a bi-dimensional model for each of the $\left(\begin{array}{l}D \\ 2\end{array}\right)$ pairwise combinations of dimensions through a pairwise composite likelihood framework. The total number of possible latent classes in each bi-dimensional case is 9 , and therefore tractable for typical genomic datasets. For each pair of dimensions, we estimate which subset of the 9 possible 


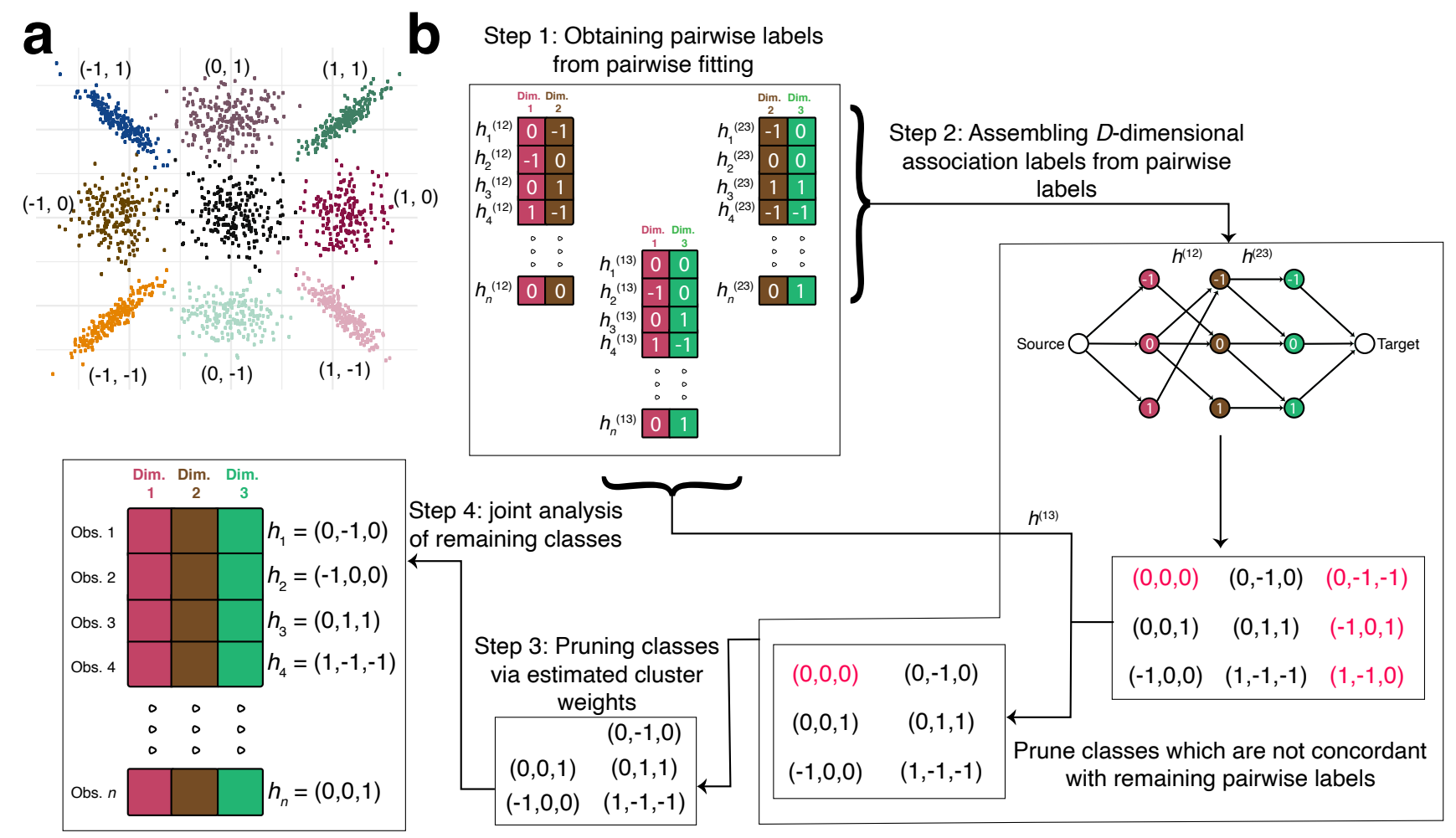

Figure 1: Toy examples of CLIMB. a, Illustration of the considered model using a simulated dataset with two dimensions. The 9 classes are annotated by their corresponding latent association vectors. The null class $(0,0)$ lies in the center over the origin. Classes that are non-null in at least one dimension exhibit a location shift. Only observations from classes that are non-null in both dimensions are correlated. b, Flowchart of CLIMB with a 3-dimensional example, with true classes whose association vectors are denoted $h_{1}, h_{2}, h_{3}, h_{4}$, and $h_{n}$. Step 1 fits 3 pairwise models. Pairwise association vectors are estimated for each observation in each pairwise fit. In Step 2, we enumerate candidate 3-dimensional association vectors using a graph-based algorithm based on the estimated pairwise association vectors (shown as edges) between dimensions 1 and 2, and the estimated pairwise association vectors between dimensions 2 and 3. 9 candidate association vectors are found on the graph, but those that are colored in red are not truly present in the data. Association vectors that are not concordant with estimated association vectors from the pairwise fit between dimensions 1 and 3 are pruned. With 6 remaining candidates, one computes their prior weights (Step 3), then in Step 4 fits a Bayesian mixture model to the original, 3-dimensional data using the number of classes remaining after Step 3.

configurations of the latent association vector are supported by the data across those 2 dimensions by utilizing a penalized mixture model ${ }^{19}$. This mixture model penalizes the class mixing weights, such that classes that are likely without members are removed from the pairwise model. Unlike many composite likelihood approaches that assume independence across dimensions ${ }^{15,20}$, the pairwise model takes account of dependence between each pair of conditions.

2. Assembling D-dimensional association labels from pairwise labels. Use the estimated pairwise 
association vectors to assemble a preliminary list of feasible $D$-dimensional association vectors. $D$-dimensional association vectors that are inconsistent with inferred pairwise labels will be deemed infeasible and pruned.

3. Pruning association labels with insufficient cluster weights. Estimate the mixing weights for the remaining latent classes using the estimates obtained from the pairwise fits, pruning classes with insufficient weight and ensuring that $M \leq n$.

4. Empirical Bayesian estimation of the full D-dimensional model. Reestimate parameters for the $D$-dimensional mixture model based on the final list of classes using a Bayesian approach. Inform prior hyperparameters with parameter estimates obtained from the pairwise fits. This final step ensures information across all dimensions is considered.

CLIMB's model output is useful for a host of analyses, including: (1) using association labels and class membership to elucidate condition-specificity, (2) using class membership probabilities to test for consistency in signals across conditions, (3) using estimated cluster covariances to infer similarity between conditions, and (4) using estimated cluster means to obtain a parsimonious characterization of dominant patterns of condition-specificy. See Methods and supplement for details on these downstream analyses.

\section{Simulations}

We used simulations to compare CLIMB to the available methods for multiconditional analysis, Urbut et al.'s mash ${ }^{14}$ and Amar et al.'s SCREEN ${ }^{16}$. We selected these two methods to compare against because they are also designed to analyze many conditions for obtaining information on condition specificity. We consider two scenarios that are commonly encountered in genomic analyses: ChIP-seq data and the differential analysis of RNA-seq data. The former simulation aimed to study cell type-specificity of patterns of protein binding across different cell types (motivating context 1), while the latter aimed to identify which genes are dysregulated in a consistent manner across different diseased tissues when compared against normal tissues (motivating context 2). These datasets exhibit different distributional structures: All signals in simulation 1 have a positive sign (e.g., see Supplementary Fig. S1a), but the signals in simulation 2 can be positive or negative. The strictly positive nature of signals in simulation 1 arises from the fact that identified protein binding sites from ChIP-seq data are output from a peak-calling routine, where each signal indicates evidence for the presence of a ChIP-seq peak at a given genomic location. In contrast, the data in simulation 2 are derived from $P$-values that indicate whether genes are relatively over- or under-expressed in a diseased tissue relative to a normal counterpart tissue. This translates to $Z$-scores exhibiting both positive and negative signals, and data that are more symmetrically distributed about the origin (e.g., see Supplementary Fig. S1b). A unifying goal of both simulations is to evaluate the capacity of all methods to adapt to data types with different distributions. See Testing consistency of effects for description of statistical test used; see Comparison against SCREEN and mash and supplement for further details on the simulation procedure.

As shown in Supplementary Tables S1-S2 and Figures S2-S6, CLIMB uniformly performed the best in both simulations across several quantitative metrics, including sensitivity and precision, indicating that CLIMB is well-suited for identifying patterns of association in the data as well as consistent signals. 

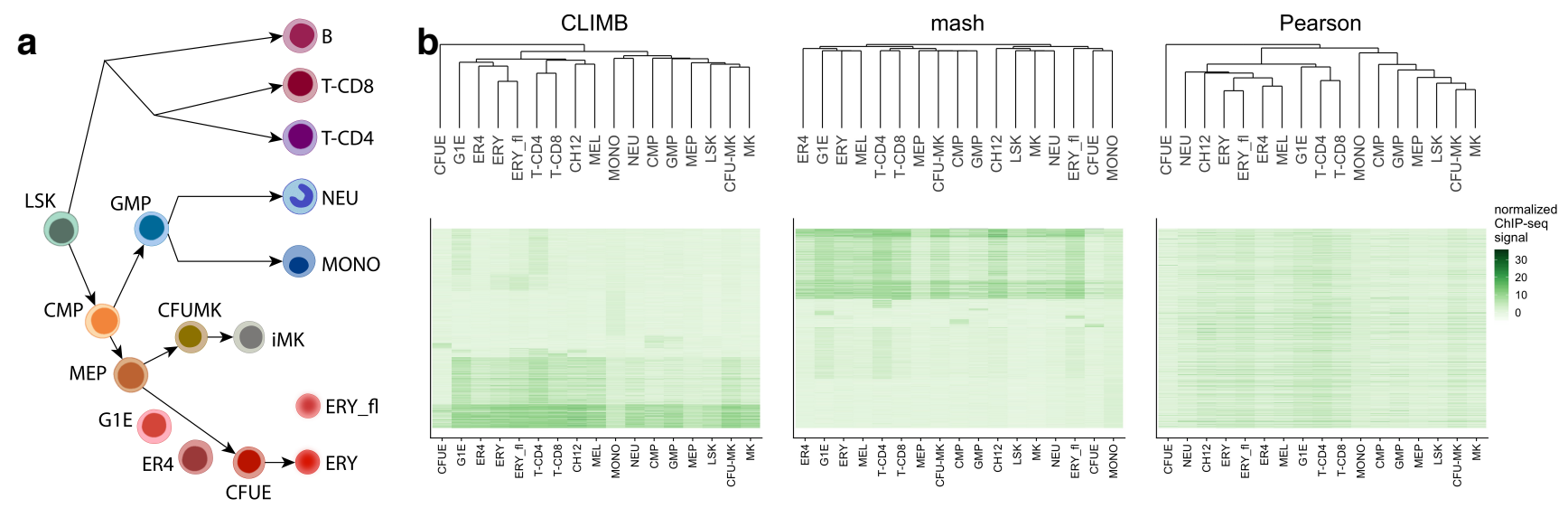

Figure 2: CLIMB uncovers interrelationships among hematopoietic cell populations based on CTCF binding patterns. a, Expected relationship among cell populations. b, Heatmaps displaying bi-clusterings of all ChIP-seq data for chromosome 11 based on CLIMB, mash, and Pearson correlation. The columns, corresponding to different cell populations, are ordered according to the dendrogram for each clustering method. The rows, corresponding to each loci, are ordered based on class membership (for CLIMB and mash) and Pearson correlation (for Pearson), respectively. (CH12 and MEL are murine lymphoma and erythroleukemia cell lines, respectively, and thus do not clearly occupy one space in the lineage, though CH12 is most related to B cells, and MEL is a mature erythroid cell type.)

\section{Case studies}

We showcase CLIMB's utility by analyzing multiple datasets collected as part of the VISION project (ValIdated Systematic IntegratiON of hematopoietic epigenomes) ${ }^{21,22,23}$. The VISION data were collected from 17 murine hematopoietic cell populations across differentiation. The primary goal of the VISION project is to understand the interplay between transcriptomic variation and mechanisms of gene regulation during hematopoiesis.

First, we study CTCF ChIP-seq data in 17 hematopoietic cell populations ${ }^{24}$. While CTCF binding sites that are invariant across cell types are known to maintain chromatin structures ${ }^{25}$, the function of more cell type-specific CTCF binding sites remains largely unknown ${ }^{5,26,27}$. We show how CLIMB can be used to aid in tackling this question. Next, we examine RNA-seq data collected from a subset of these cell populations to probe the transcriptomic changes that commit multipotent cells to different fates. Results from these analyses demonstrate CLIMB's ability to elucidate interrelationships between cell populations in different genomic data types, produce interpretable classes, and conduct lineage-specific differential analyses.

\section{VISION CTCF ChIP-seq}

We applied CLIMB to CTCF ChIP-seq of chromosome 11 from 17 murine cell populations. This analysis yielded a final model that included 15 non-empty classes. Among these, 2 classes described constitutive binding behavior, while the remaining were more cell type-specific (see Supplementary Fig. S7 for an illustration of all classes). Similar results are obtained for chromosome 7 (see Supplementary Section Analysis of CTCF ChIP-seq on chromosome 7). 


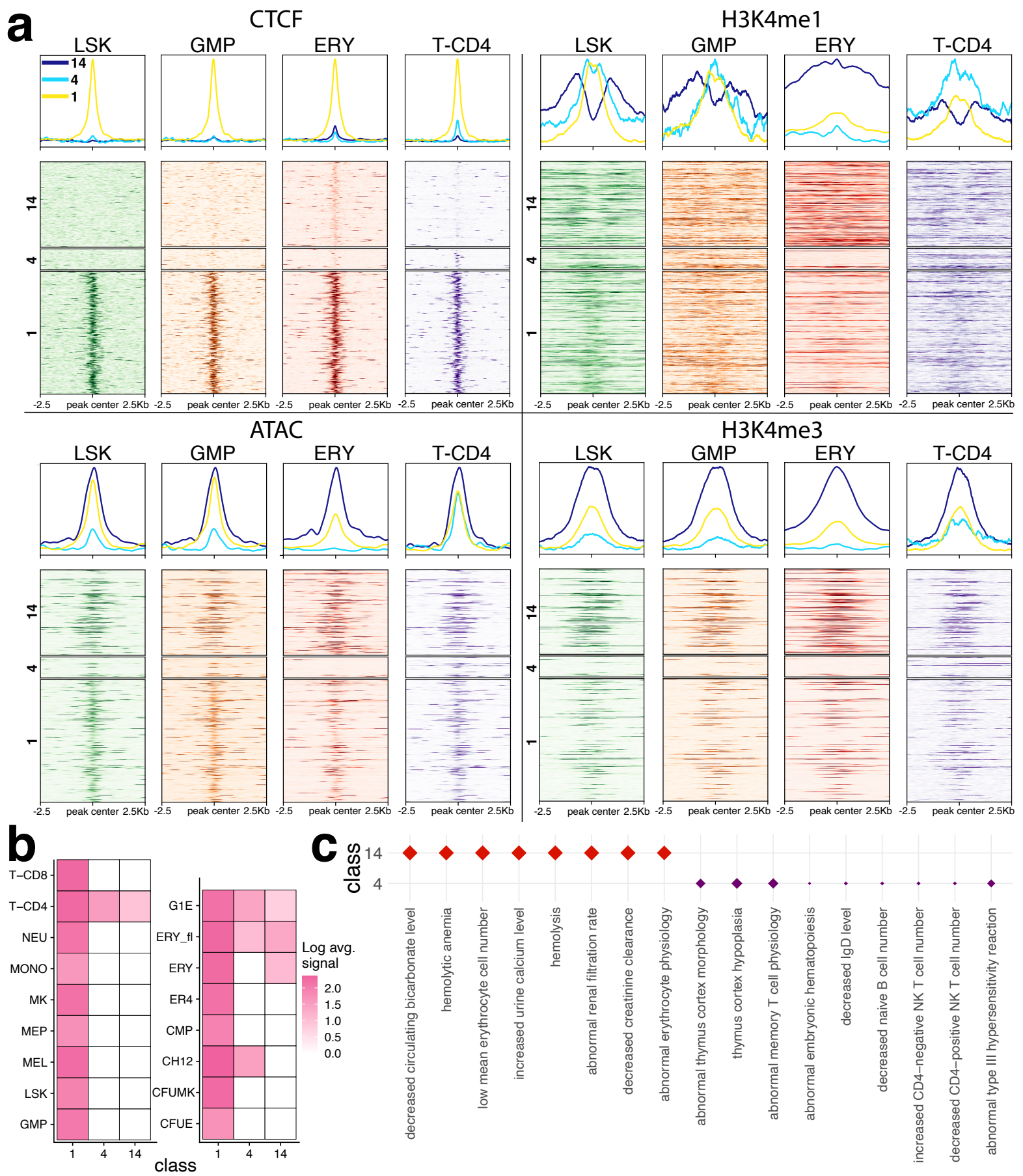

Figure 3: CTCF binding patterns uncovered by CLIMB capture different patterns of epigenetic modifications. a, Data from the loci on chromosome 11 that belong to classes of CTCF binding patterns (numbered 1, 4, and 14) identified by CLIMB are shown. The original CTCF ChIP-seq, alongside ATAC-seq and histone modification ChIP-seq data in 4 hematopoietic cell populations reveal differing patterns of epigenetic modifications across cell populations. b, Log class means based on CLIMB's model of CTCF binding patterns for the 3 classes in a. c, Significantly enriched mouse phenotypes (FDR $<0.05$ for all) associated with the plotted classes. Class 1, containing loci with CTCF bound in every cell type, is not significantly enriched in any mouse phenotypes. Class 4 is enriched with terms related to $\mathrm{T}$ and $\mathrm{B}$ cells and the thymus, while class 14 contains terms related to red blood cells and kidney function. 
Constitutively bound CTCF is the dominant class. Previous work has noted that CTCF binding is largely consistent across cell types ${ }^{5,25,28}$. We identified two such classes of conserved loci from CLIMB's model fit. The first is the class of all ones, corresponding to the collection of loci bound by CTCF across all cell types. The second is the class of all ones except for the CFUE population, corresponding to the collection of loci bound by CTCF in all but the CFUE cell population, likely reflecting lower signal-to-noise ratio in the CFUE dataset. Indeed, the CFUE experiment had the lowest quality as measured by FRiP score ${ }^{29}$ (0.031, compared against next lowest iMK with FRiP score 0.054 and CMP with FRiP score 0.097). In agreement with previous studies, these two classes make up $\sim 36 \%$ of all loci in the analysis. Moreover, consistent with others ${ }^{28,30}$, the average signal strength (based on the estimated class means) for bound loci within the two constitutive classes is significantly larger than the average signal strength for bound loci that are not widely shared across cell populations (one-sided $t$-test, $P=5.02 \times 10^{-12}$ ).

Differential CTCF binding is predictive of cell population relationships. Although CTCF binding is largely consistent across cell types, previous studies suggested that changes in its binding patterns modify gene expression programs, affecting developmental cues or cell function ${ }^{5,30,31}$. We asked whether the classes discovered by CLIMB support the idea that changes in CTCF binding relate to hematopoietic development. To address this, we clustered the cell populations based on the estimated class covariance matrices ${ }^{32}$ (see supplementary Implementation details). CLIMB's clustering, shown in Fig. 2b, closely reflects the expected lineage relationship in Fig. 2a. This result supports the claim that changes in CTCF binding occur in a lineage-specific manner, and that CLIMB is well-suited to tease out this information from the data. In contrast, the clusterings based on mash and the standard hierarchical clustering using Pearson correlation are quite different from the expected lineage relationship (e.g., mash places CFU-MK and MK cells far from one another on the hierarchical tree, and both mash and the standard hierarchical clustering place MONO and NEU cells far from one another on the hierarchical tree). This suggests that mash does not sufficiently capture CTCF binding patterns across cell types, and that simple correlation measures cannot uncover heterogeneous signals in the data. The low signal in the CFUE experiment likely caused the hierarchical clusterings by both CLIMB and Pearson correlation to isolate the CFUE cell from the remaining cell populations on the hierarchical tree. CLIMB exhibits robustness to this challenge, identifying this cell as an outlier among all experiments, while still achieving a hierarchical clustering that reflects the expected relationship among the remaining cell populations.

CLIMB identifies succinct groupings of CTCF binding patterns. Visualization of genomic loci assigned to different classes is important for identifying biologically meaningful patterns. To facilitate visual examination, CLIMB provides a means to merge similar classes based on model output (see supplementary Implementation details for details on the class merging procedure). From the VISION CTCF dataset, CLIMB clusters the binding sites into 15 non-empty classes. To simplify the visualization, we merged these classes into 5 parent groups, with sizes ranging from 254 to 5,462 loci. Supplementary Fig. S8a displays the average signal strength (Equation 30) associated with each of these groups. For example, group 1 includes constitutive binding sites, while group 4 contains progenitor-specific binding sites, and group 5 contains binding sites constituent to mature erythroid and T cells. Supplementary Fig. S8b displays the locations of the binding groups within the genomic region around murine gene $B c l 11 a$, whose gene product is involved in gene regulation 
of multiple cell types.

CTCF binding patterns relate to epigenetic states during differentiation We next examined how CLIMB's classes of CTCF binding patterns relate to chromatin accessibility and various histone modifications. Interestingly, though we only supplied CTCF ChIP-seq data to each method, the classes estimated by CLIMB also displayed cell type-specific behavior of chromatin accessibility as measured using ATAC-seq and epigenetic histone modifications H3K4me1 and H3K4me3 (Fig. 3ab). Further, using GREAT ${ }^{33}$ (Genomic Regions Enrichment of Annotations Tool), we identified that classes that exhibit erythroid- and immune cell-specific binding patterns are indeed enriched in erythroid- and T cell-specific functions (Fig. 3c). In contrast, the classes identified by mash do not appear to relate to epigenetic modifications (Supplementary Figures S9-S12). In fact, there is not a large amount of overlap between CLIMB's and mash's estimated classes (Supplementary Fig. S13), altogether suggesting that CLIMB effectively captures biologically meaningful protein binding patterns.

\section{VISION RNA-seq}

We next used CLIMB to perform lineage-specific differential expression analysis. In the hematopoietic cell system, LSK, CMP and MEP are multipotent cells that differentiate into different terminal cells, such as ERY, MONO, NEU, and iMK cells (Fig. 2a). We considered three paths: the erythroid lineage $(\mathrm{LSK} \rightarrow \mathrm{CMP} \rightarrow \mathrm{MEP} \rightarrow \mathrm{CFUE} \rightarrow \mathrm{ERY})$, the megakaryocytic lineage $(\mathrm{LSK} \rightarrow \mathrm{CMP} \rightarrow$ $\mathrm{MEP} \rightarrow \mathrm{CFUMK} \rightarrow \mathrm{iMK}$ ), and the myeloid lineage (LSK $\rightarrow \mathrm{CMP} \rightarrow \mathrm{GMP} \rightarrow \mathrm{MONO} / \mathrm{NEU})$. The differentially expressed genes identified in each linage are expected to be related to the biological function of the specific differentiation path and cell fate commitment. The datasets for these lineages respectively contained 21,303, 20,995, and 22,940 expressed genes.

CLIMB identifies lineage-specific genes related to cell development and differentiation. We sought to identify genes that show varying gene expression levels across each differentiation path. We first fit a model with CLIMB to each lineage (see Supplementary Fig. S16 for an illustration of estimated classes for each lineage) and then pinpointed the genes that exhibit differential signals across each lineage based on model fit. To proceed, we first identified genes with consistent signals by performing a statistical test (see Methods). Briefly, a gene was considered "consistently expressed" across the lineage if its probability of belonging to a class that is interpreted as describing consistent expression behavior is sufficiently large. These classes are: $(-1,-1,-1,-1,-1),(0,0,0,0,0)$, or $(1,1,1,1,1)$, where $h_{[d]}=-1$ implies a gene is lowly expressed or off, $h_{[d]}=0$ implies a gene is moderately expressed, and $h_{[d]}=1$ implies a gene is highly expressed in cell population $d$. Otherwise, a gene was considered differentially expressed (DE) along the lineage. Our results identified 2,242 DE genes along the erythroid lineage (including erythroid marker genes Alas2, Hba-a1, Hba-a2, and Gata1), 2,073 along the megakaryocytic lineage (including megakaryocytic marker genes Itgb3, Ltb4r1, Il3ra, and Il6ra), and 2,376 along the myeloid lineage (including myeloid marker genes Cxcr2, C5ar1, S100a8, and S100a9). A Venn diagram displaying the overlap of DE genes across lineages is in Supplementary Fig. S17.

Alternatively, a common approach to this sort of problem is to apply a series of pairwise differential expression analyses along each lineage with standard software such as DESeq2 ${ }^{34}$, then 


\begin{tabular}{llr}
\hline Lineage & Gene ontology term & Raw $P$-value \\
\hline erythroid & heme biosynthetic process & $1.91 \times 10^{-4}$ \\
& heme metabolic process & $2.88 \times 10^{-5}$ \\
& erythrocyte differentiation & $2.27 \times 10^{-4}$ \\
& erythrocyte homeostasis & $6.76 \times 10^{-5}$ \\
\hline \multirow{2}{*}{ megakaryocytic } & regulation of platelet activation & $1.67 \times 10^{-4}$ \\
& regulation of blood coagulation & $2.68 \times 10^{-6}$ \\
& response to wounding & $1.43 \times 10^{-6}$ \\
& regulation of homotypic cell-cell adhesion & $1.58 \times 10^{-3}$ \\
\hline \multirow{2}{*}{ myeloid } & regulation of monocyte chemotaxis & $6.82 \times 10^{-4}$ \\
& positive regulation of leukocyte degranulation & $2.25 \times 10^{-3}$ \\
& positive regulation of neutrophil migration & $9.68 \times 10^{-5}$ \\
& regulation of macrophage activation & $2.23 \times 10^{-5}$ \\
\hline
\end{tabular}

Table 1: Lineage-specific differentially expressed genes identified by CLIMB are enriched in gene ontology terms related to terminal cell function.

take the union of all DE genes across the analyses. We implemented this strategy using DESeq2 with FDR $\leq 0.01$ and obtained $6,883 \mathrm{DE}$ genes across the erythroid lineage, 7,458 across the megakaryocytic lineage, and 6,863 across the myeloid lineage. The number of DE genes called by DESeq2 was about one third of all input genes for each analysis, and about 3 times more than the number of DE genes identified by CLIMB. We also applied SCREEN to identify DE genes along each lineage, and found that SCREEN systematically underperformed relative to both CLIMB and DESeq2 (Supplementary Fig. S18).

The large number of DE genes returned by DESeq2 raises questions about the specificity of this approach in pinpointing genes relevant to differentiation. To probe whether DESeq2 is exhibiting low precision or CLIMB exhibiting low power, we ran gene ontology (GO) enrichment analyses for each lineage ${ }^{35,36}$. Some enriched GO terms from the CLIMB analysis of each lineage are in Table 1. Meanwhile, the DESeq2 gene set was not enriched in hematopoiesis-specific GO terms. The abundance of CLIMB's enriched hematopoiesis-specific GO terms suggests that, though CLIMB identifies far fewer DE genes than DESeq2, CLIMB identifies more key genes relevant to cell development and differentiation.

To more directly compare CLIMB and DESeq2, we partitioned DE genes into three categories, namely, differentially expressed genes specific to CLIMB, DE genes specific to DESeq2, and DE genes in the intersection of both methods for each lineage (Fig. 4a), and ran GO analyses on these sets. We noticed that genes identified as DE by both CLIMB and DESeq2 are enriched in many hematopoietic-related terms, while DESeq2-specific genes are enriched for many terms related to general cell function. In each lineage, DESeq2-specific genes are highly enriched for functions that are not specific to hematopoietic cells; CLIMB-specific genes in general are not highly enriched for these same terms. Both genes identified by both CLIMB and DESeq2 and CLIMB-specific genes are more frequently enriched for hematopoietic-specific functions (Fig. 4b). The result that DESeq2's significant gene sets are only enriched in hematopoiesis-related GO terms 


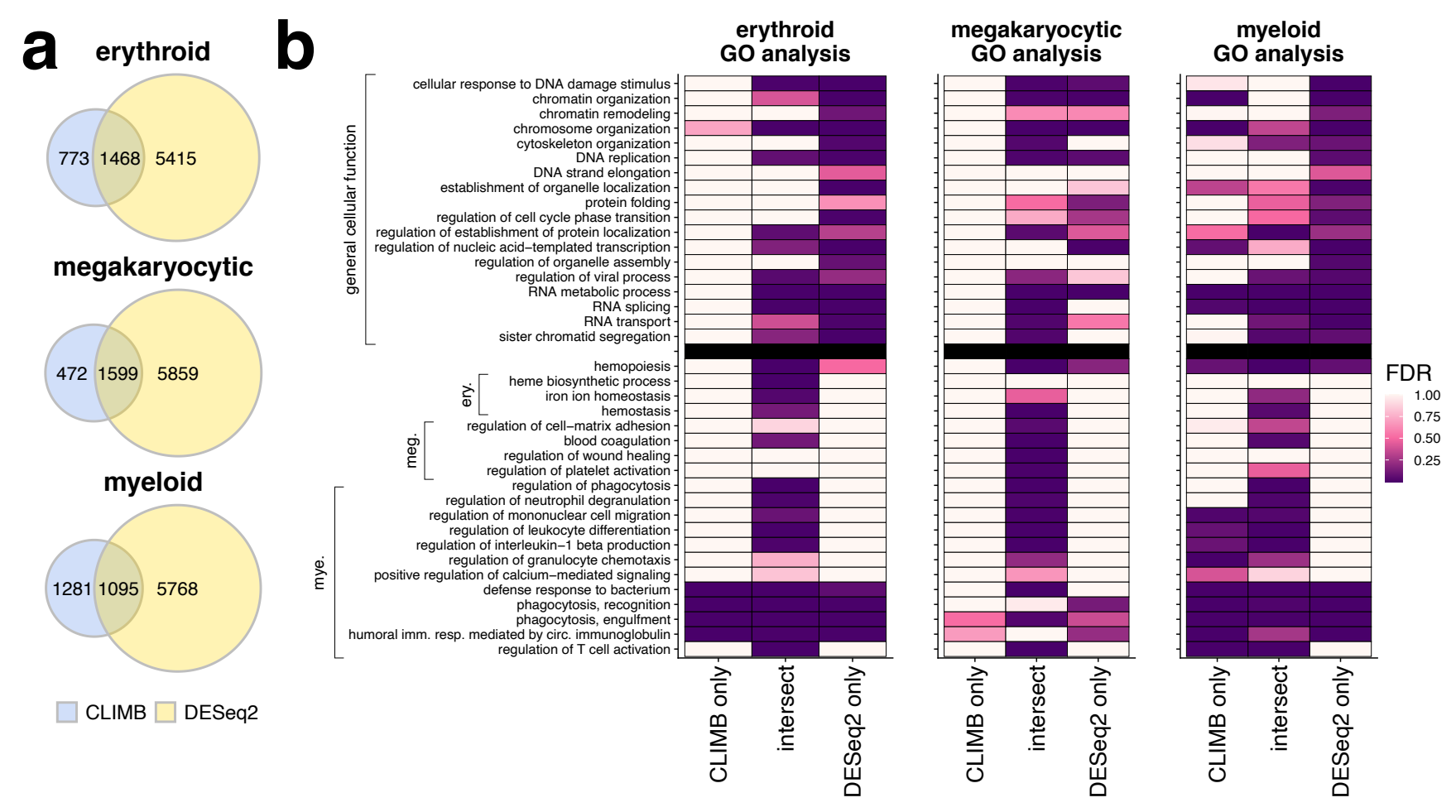

Figure 4: Comparison of differentially expressed genes identified by CLIMB and DESeq2. a, Venn diagrams displaying overlap of differentially expressed genes identified by both methods across all analyses. b, Significance of enrichment of GO terms in gene sets specific to CLIMB, specific to DESeq2, and in the intersection of both methods, for each studied lineage. Presented GO terms are organized according to knowledge-driven labels. Non-hematopoietic terms related to general cell function are above the black line. Hematopoietic-related terms, grouped according to lineage-specific function, are below the black line.

after intersection with CLIMB's significant gene sets demonstrates that CLIMB is a powerful and more precise approach to multi-condition differential gene expression analysis when compared to DESeq2 applied in a series across multiple conditions.

CLIMB latent association labels describe patterns of expression across cell differentiation. Next we used CLIMB to further probe specific gene expression patterns of interest. For example, in the erythroid analysis, 559 genes fell into the $(-1,-1,-1,1,1)$ class. This class describes genes with little to no expression in the LSK, CMP, and MEP cell populations, but high expression in the CFUE and ERY cell populations. This gene set is enriched for GO terms such as erythrocyte development $\left(P=3.09 \times 10^{-9}\right)$, iron ion homeostasis $\left(P=6.89 \times 10^{-5}\right)$, and humoral immune response mediated by circulating immunoglobulin $\left(P=1.07 \times 10^{-6}\right)$. Cases of enrichment for terms related to other cell types may result from a process initially discovered in the other cell type being present also in the cell type of interest.

As another example, the 298 members of the $(0,0,0,-1,-1)$ class from the myeloid lineage, corresponding to genes that are moderately expressed in LSK, CMP, and GMP cell populations, but lowly or not expressed in monocyte and neutrophil cell populations, are enriched for several 
GO terms concerning cell fate determination, such as microtubule cytoskeleton organization $\left(P=1.36 \times 10^{-8}\right)$ and mitotic cell cycle process $\left(P=2.24 \times 10^{-15}\right)$. Meanwhile, the 474 members of the $(-1,-1,-1,-1,0)$ class, corresponding to moderate gene expression specific to neutrophils, are enriched for GO terms immunoglobulin mediated immune response $\left(P=4.33 \times 10^{-23}\right)$, defense response to bacterium $\left(P=2.16 \times 10^{-22}\right)$, and immune response-activating signal transduction $\left(P=9.21 \times 10^{-29}\right)$. Moreover, the 777 members of the $(-1,-1,-1,0,-1)$ class, corresponding to genes exhibiting moderate expression specific to monocytes, are enriched for the GO terms for the production of tumor necrosis factor and interleukins 1, 6, and 12, as well as the regulation of mast cell activation $\left(P=7.14 \times 10^{-4}\right)$. Taken together, these results demonstrate that CLIMB's utility goes beyond lineage-specific differential gene expression analysis; the individual latent classes also describe interpretable gene expression patterns.

\section{Discussion}

We present a new method, CLIMB, for joint analysis of genomic data collected from multiple experimental conditions. CLIMB gains statistical power to uncover biologically relevant signals by providing a means to extend typical pairwise analyses to higher dimensions. Moreover, when compared against methods designed for a higher-dimensional setting, we demonstrated that CLIMB remains powerful, flexible, and interpretable in many contexts. Since CLIMB's mixture modeling framework is quite flexible, it is effective on a wide range of input data, as long as the data can be reported as numerical scores that reflect the strength of association.

A major benefit of CLIMB is its ability to describe various patterns of condition-specificty in a mixture with corresponding association vectors that are estimated from the data. The model, aided by these association vectors, is scientifically interpretable. Estimated model parameters can elucidate similarity and interrelationships, and parsimoniously characterize representative association patterns present across experimental conditions. Importantly, the association vectors also serve as the basis for a novel and effective means of testing consistency of signals across several conditions or biological experiments.

\section{Methods}

\section{Constrained mixture model for estimating association vectors}

Recall that to estimate the association vectors, we consider the following mixture model. Define

$$
\begin{aligned}
n & :=\text { number of observations, } \\
D & :=\text { dimension of data } \\
H=\left(h_{[1]}, \ldots, h_{[D]}\right) & :=\text { latent association vector } \\
h_{[d]} & \in\{-1,0,1\}, d \in\{1, \ldots, D\},
\end{aligned}
$$

such that the observed data follow the constrained normal mixture model 


$$
\begin{aligned}
\mathbf{x} \mid H=h_{m} & \sim \phi_{D}^{c}\left(\boldsymbol{\mu}_{m}, \boldsymbol{\Sigma}_{m}, h_{m}\right) \\
H & \sim \operatorname{Mult}\left(\pi_{1}, \ldots, \pi_{M}\right), \quad \sum_{m=1}^{M} \pi_{m}=1
\end{aligned}
$$

where $h_{m}$ is the $m^{\text {th }}$ latent class, $m \in 1, \ldots, M$, and $\phi_{D}^{c}$ is a $D$-dimensional constrained normal distribution. Note that the number of candidate latent classes $M$ changes as our methodology prunes unsupported classes (see Pairwise fitting and subsequent methodological steps).

If an observation has association label $h_{[d]}=1\left(h_{[d]}=-1\right)$, this implies that it exhibits a significant positive (negative) association with condition $d$. Otherwise, if an observations has association label $h_{[d]}=0$, this implied that it exhibits a null association with condition $d$. To capture this relationship described by the association vectors, we set the following constrains on $\phi_{D}^{c}$ :

1. Null associations in dimension $d$ are assumed to follow the standard normal distribution $\left(\mu_{d}=0, \sigma_{d}=1\right)$.

2. Non-nulls that have a positive (negative) association in dimension $d$ have a strictly positive (negative) mean in dimension $d$.

3. Nulls in one dimension do not correlate with non-null associations in any other dimension $\left(\Sigma_{r t}=0 \forall t \neq r\right.$ if either $h_{[r]}=0$ or $\left.h_{[t]}=0\right)$.

4. Non-nulls that show concordant (discordant) associations across dimensions - i.e., $h_{[r]}=h_{[t]}$ $\left(h_{[r]}=-h_{[t]}\right)$ where $h_{[r]} \in\{-1,1\}$-are positively (negatively) correlated, that is, $\Sigma_{r t}>0$ $\left(\Sigma_{r t}<0\right)$.

A 2-dimensional visualization of these constraints is in Fig. 1a. Though these constraints are desirable for interpretability, imposing them through latent association vectors leads to computational difficulties as the number of dimensions grows because there are $3^{D}$ possible configurations of the latent association vectors. We thus developed CLIMB, a modeling strategy designed to circumvent the computational intractability that arises under these circumstances. We now describe the steps of CLIMB in greater detail.

\section{Detailed CLIMB procedure}

\section{Pairwise fitting}

Composite likelihood (CL) methods ${ }^{37}$, which have been reviewed extensively ${ }^{38}$, are computationally efficient modeling approaches that approximate the joint data model by making certain conditional independence assumptions. CL methods are frequently utilized in statistical literature. For instance, they can simplify a genetic model of recombination rates by assuming conditional independence given nearest neighbors along the genome ${ }^{39}$, or sidestep specifying a complex joint likelihood in favor of a product of bivariate models ${ }^{40}$. CL estimators are consistent, though they exhibit some loss in efficiency. 
We are seeking to reduce model complexity in the number of latent classes by limiting the dimension of the data through pairwise CL. Let $\Omega=\left\{\left(\mathbf{X}_{\cdot 1}, \mathbf{X}_{\cdot 2}\right), \ldots,\left(\mathbf{X}_{\cdot D-1}, \mathbf{X}_{\cdot D}\right)\right\}$ be the set of all pairs of dimensions of $\mathbf{X}_{n \times D}$, giving $|\Omega|=\left(\begin{array}{c}D \\ 2\end{array}\right)$. The pairwise CL is

$$
\begin{aligned}
\mathcal{L}_{C}(\boldsymbol{\theta}): & =\mathcal{L}_{C}\left(\mathbf{X}_{\cdot 1}, \ldots, \mathbf{X}_{\cdot D} \mid \boldsymbol{\theta}\right) \\
& =\prod_{r=1}^{D-1} \prod_{t=r+1}^{D} f_{r t}(\cdot \mid \boldsymbol{\theta}) \\
& =\prod_{r=1}^{D-1} \prod_{t=r+1}^{D} \prod_{i=1}^{n} \sum_{m=1}^{M} \pi_{m} \phi_{2}^{c}\left(\mathbf{X}_{i, r t} \mid \boldsymbol{\theta}_{r t}, h_{m}^{(r t)}\right)
\end{aligned}
$$

where $\mathbf{X}_{\text {.rt }}$ is the $n \times 2$ matrix of observations from dimensions $r$ and $t, \boldsymbol{\theta}_{r t}$ is the parameter vector describing the normal mixture between dimensions $r$ and $t$, and $h_{m}^{(r t)}$ is the $m^{\text {th }}$ class in the set of all possible 2-dimensional latent association vectors $h_{r t}$ between dimensions $r$ and $t$. Note that for each pair in $\Omega$, each pairwise model, $f_{r t}$, is computationally tractable. This style of pairwise CL, termed "pairwise fitting", has been utilized most frequently to alleviate computational difficulty when analyzing survey data with multivariate responses ${ }^{41,42,43,44,45}$. Because each dimension appears in $D-1$ different pairwise fits, the mean and variance of each class are estimated $D-1$ times, leading to $D-1$ not necessarily equal estimates for the same mean and variance. It has been shown that, though these pairwise estimates are redundant and not necessarily concordant, they carry useful information about the true parameters ${ }^{45}$. Thus we will recycle these estimates to inform the priors in the final step of our procedure (see An empirical Bayesian model).

Fitting each pairwise model $f_{r t}$ amounts to fitting a finite normal mixture model arising from 9 classes described by latent association vectors $h \in \mathcal{H}_{r t}$ where

$$
\mathcal{H}_{r t}=\{(-1,-1),(-1,0),(-1,1),(0,-1),(0,0),(0,1),(1,-1),(1,0),(1,1)\} \quad \forall r<t .
$$

However, since the total number of latent classes in the full model is less than $3^{D}$, we expect that the true number of latent classes in some, if not all of the pairwise fits, is less than 9. Accordingly, for each pairwise fit, we perform model selection to filter out unsupported classes at the pairwise level using a previously described penalized maximum likelihood approach ${ }^{19}$. This method provides an automated model selection procedure for normal mixture models with theoretical guarantees of consistency in selecting the correct number of clusters (see Model selection details).

\section{Construction of $D$-dimensional association labels}

Next, we assemble the list of candidate $D$-dimensional latent association vectors by concatenating all the pairwise association vectors of adjacent dimensions estimated in the previous step. Only association vectors that are on this candidate list are retained for downstream analyses. Example 1 shows a simple example for a 3-dimensional dataset.

Example 1: Let $\mathcal{H}_{r t} \subseteq \mathcal{H}_{r t}$ be the set of 2-dimensional latent association vectors present in a model of dimensions $r$ and $t$. Now, consider a three-dimensional dataset, where latent association vectors $(-1,0) \in \mathcal{H}_{12}$ and $(0,1) \in \mathcal{H}_{23}$. These two association vectors suggest that some observations 
belong to the null class in dimension 2 , and that some of these observations exhibit negative signals in dimension 1 [since $(-1,0) \in \mathcal{H}_{12}$ ], and positive signals in dimension 3 [because $(0,1) \in \mathcal{H}_{23}$ ]. Thus, the data support that $(-1,0,1)$ remains a candidate $D$-dimensional latent association vector.

To perform this task computationally efficiently, we construct a directed acyclic graphical representation of the pairwise classification results, designed in the spirit of a de Bruijn graph ${ }^{46,47}$. This novel representation allows one to efficiently enumerate all plausible candidate $D$-dimensional latent association vectors in the concatenation by applying a standard graph search algorithm.

Specifically, we denote a vertex in the graph as $(d, a)$, representing a possible association, $a$, at a given dimension, $d$. For a model with $D$ dimensions, the graph has $D$ layers and 3 possible associations at each layer: $-1,0$, and 1. A pictorial view is in Supplementary Fig. S19. We write the vertex set as the collection of all ordered pairs

$$
V^{\prime}=\{(d, a): d \in\{1, \ldots, D\}, a \in\{-1,0,1\}\} .
$$

The edge set is defined as

$$
E^{\prime}=\left\{\left[\left(d, a_{1}\right),\left(d+1, a_{2}\right)\right]: d \in\{1, \ldots, D-1\}, a_{1}, a_{2} \in\{-1,0,1\},\left(a_{1}, a_{2}\right) \in \mathcal{H}_{d, d+1}\right\} .
$$

The final graph also contains dummy source and target nodes $S$ and $T$, such that the final vertex set $V=V^{\prime} \cup\{S, T\}$. The source node has edges pointing to all nodes in layer 1, while each node in layer $D$ has an edge pointing to the target node. The final edge set is then defined as

$$
E=E^{\prime} \cup\{[S,(1,-1)],[S,(1,0)],[S,(1,1)],[(D,-1), T],[(D, 0), T],[(D, 1), T]\} .
$$

Once the graph is constructed, depth-first search with backtracking ${ }^{48}$, a graph search algorithm that enumerates all paths in a graph from a given source node to a given target node, is used to enumerate all paths from $S$ to $T$. Each path contains one node from each of the $D$ layers plus the source and target nodes, and has $D+1$ edges of the form

$$
\left\{\left[S,\left(1, a_{1}\right)\right],\left[\left(1, a_{1}\right),\left(2, a_{2}\right)\right],\left[\left(2, a_{2}\right),\left(3, a_{3}\right)\right], \ldots,\left[\left(D-1, a_{D-1}\right),\left(D, a_{D}\right)\right],\left[\left(D, a_{D}\right), T\right]\right\} .
$$

This path corresponds to the latent association vector $\left(a_{1}, \ldots, a_{D}\right)$.

\section{Pairwise fit-based pruning}

The initial construction of the graph in Construction of D-dimensional association labels only uses output from the $D-1$ pairwise fits between dimensions $d$ and $d+1$ for $d \in\{1, \ldots, D-1\}$. Certain paths may be incompatible with the remaining $\left(\begin{array}{l}D \\ 2\end{array}\right)-(D-1)$ fits. We next remove these paths 
from the candidate list by checking for incompatabilities, in a manner similar to the continuation of Example 1 below.

Example 1 (continued): As shown previously, $(-1,0,1)$ was identified as a candidate $D$-dimensional latent association vector. If $(-1,1) \notin \mathcal{H}_{13}$, then the latent class $(-1,0,1)$ is discarded from downstream analysis. This is because $\mathcal{H}_{13}$ shows that $(-1,0,1)$ is incompatible with the pairwise findings.

The graph-based enumeration and pruning algorithm is a deterministic procedure that is guaranteed to produce a list of candidate latent classes that includes all true underlying classes with the possibility of additional empty classes, assuming the correct pairwise classes were estimated (Proposition 1). Further, the results are not affected by reordering of the dimensions (Proposition 2, see Supplementary Section 1 for formal proofs).

\section{Mixing weight-based class pruning}

Since the pairwise fit-based class pruning procedure is conservative, some remaining candidate classes still may not be present in the data (e.g, the $(0,0,0)$ latent association label in the toy example in Fig. 1). To prune these classes, we estimate the weights of the remaining classes based on the pairwise fitting, and remove those whose weights are near zero. To elucidate which classes are unsupported, we devise an estimator that measures the concordance between the candidate list of $D$-dimensional association labels against the pairwise labels for each observation. Intuitively, our estimator is motivated by the assertion that if observation $\mathbf{x}$ belongs to a given class $h$, then x's pairwise latent class assignment $h^{(r t)}$ should equal $\left(h_{[r]}, h_{[t]}\right)$ for most pairs $r$ and $t, r<t$. Then, the weight for a $D$-dimensional class can be estimated by computing the proportion of observations that follow the pairwise labels of the D-dimensional association vector closely.

To construct such an estimator, let $\mathbf{x}_{i}^{(r t)}$ be the sub-vector of the $i^{\text {th }}$ observation vector corresponding to the pairwise fit between dimensions $r$ and $t$. Then, let $H_{i}^{(r t)}$ be the pairwise association vector assigned to observation $\mathbf{x}_{i}^{(r t)}$. Assuming there are $M$ remaining candidate $D$-dimensional latent classes $h_{m}, m \in\{1, \ldots, M\}$, let $h_{m}^{(r t)}$ be the sub-vector of $h_{m}$ corresponding to dimensions $r$ and $t$. Then, for a given $D$-dimensional latent class $h_{m}$, define

$$
\hat{\alpha}_{m}=\frac{\sum_{i=1}^{n} \mathbb{1}\left\{\left[\sum_{r<t} \mathbb{1}\left(H_{i}^{(r t)}=h_{m}^{(r t)}\right)\right] \geq\left(\begin{array}{c}
D \\
2
\end{array}\right)-\delta\right\}}{\sum_{m^{\prime}=1}^{M} \sum_{i=1}^{n} \mathbb{1}\left\{\left[\sum_{r<t} \mathbb{1}\left(H_{i}^{(r t)}=h_{m^{\prime}}^{(r t)}\right)\right] \geq\left(\begin{array}{c}
D \\
2
\end{array}\right)-\delta\right\}}
$$

as the normalized proportion of observations whose pairwise class labels are concordant, up to tolerance $\delta$, with $h_{m}$, where $\delta \in\left\{0,1, \ldots,\left(\begin{array}{c}D \\ 2\end{array}\right)\right\}$, which controls the permitted level of discordance between an observation's pairwise class labels and its $D$-dimensional latent class. We show that $\hat{\boldsymbol{\alpha}}$ is a reasonable estimator of the proportion of observations belonging to each class $h_{m}$ given the data (see Proofs, Proposition 3).

When the list of remaining candidate latent classes is still large, even after the previous pruning steps in previous section, $\hat{\alpha}_{m}$ may be very close or exactly equal to 0 for many $m$. This step will remove these classes, guaranteeing that the number of remaining classes $M$ is bounded above by 
the sample size $n$. In practice, we find that this procedure often can reduce $M$ to be less than $0.01 n$.

To estimate $\hat{\alpha}_{m}$, we first obtain each $H_{i}^{(r t)}$ by sampling the pairwise labels of the $\mathbf{x}_{i}$ 's according to their posterior probabilities of belonging to each class estimated from the pairwise fits:

$$
H_{i}^{(r t)} \sim \text { Categorical }\left(\hat{p}_{1}, \ldots, \hat{p}_{M^{(r t)}}\right)
$$

where $\hat{p}_{m}=\operatorname{Pr}\left[\mathbf{x}_{i}^{(r t)} \in h_{m}^{(r t)}\right]$, the estimated posterior probability that observation $\mathbf{x}_{i}^{(r t)}$ belongs to class $h_{m}^{(r t)}$ for $m \in\left\{1, \ldots M^{(r t)}\right\}$, and $M^{(r t)}$ is the number of pairwise latent classes estimated to be present in pairwise fit between dimensions $r$ and $t$. Because $\hat{\boldsymbol{\alpha}}:=\left\{\hat{\alpha}_{1}, \ldots, \hat{\alpha}_{M}\right\}$ estimates the proportion of observations belonging to each class $h_{m}, m=1, \ldots, M$, we treat $\hat{\boldsymbol{\alpha}}$ as the prior probabilities for the class mixing weights in the $D$-dimensional model in the next and final step of CLIMB (see next section).

The number of observations needed to obtain a good estimate $\hat{\boldsymbol{\alpha}}$ is affected both by the dimension of the data and the accuracy of estimates made during pairwise fitting. For datasets with well-separated clusters, a more stringent $\delta$ (i.e. $\delta<.15 \times\left(\begin{array}{l}D \\ 2\end{array}\right)$ ) is recommended, whereas a relaxed $\delta$ (i.e. $\delta \in\left[.15 \times\left(\begin{array}{c}D \\ 2\end{array}\right), .30 \times\left(\begin{array}{c}D \\ 2\end{array}\right)\right]$ ) is more suited for datasets with less separated clusters to avoid removing true classes that are small in size. This heuristic guide may be refined by then selecting $\delta$ within this range where $M$ remains constant for $\delta^{\prime} \in\{\delta, \delta+1, \ldots, \delta+c\}$ for some $c \geq 1$. While this step of our methodology requires user input, it requires similar levels of user input as in existing methods.

\section{An empirical Bayesian model}

With the steps described thus far, we are able to pare down the number of latent classes to a more computationally manageable size for regular mixture modeling. Next we reestimate the parameters in the $D$-dimensional model (1) using an empirical Bayesian approach, recycling the pairwise estimates as prior hyperparameters. We employ the following hierarchical structure to represent the constrained mixture model:

$$
\begin{aligned}
\mathbf{x}_{i} \mid \boldsymbol{\mu}_{h}, \Sigma_{h}, H_{i}=h & \sim \phi_{D}^{c}\left(\boldsymbol{\mu}_{h}, \Sigma_{h}, h\right) \\
\boldsymbol{\mu}_{h} \mid \Sigma_{h}, H_{i}=h & \sim \phi_{D}\left(\boldsymbol{\mu}_{h}^{0}, \Sigma_{h} / \kappa_{h}\right) \\
\Sigma_{h} \mid H_{i}=h & \sim \mathcal{I} \mathcal{W}_{D}\left(\Psi_{h}^{0}, \nu_{h}\right) \\
H_{i} \mid \boldsymbol{\pi} & \sim \operatorname{Mult}(\boldsymbol{\pi}) \\
\boldsymbol{\pi} & \sim \operatorname{Dir}(\boldsymbol{\alpha})
\end{aligned}
$$

Quantities $\boldsymbol{\mu}_{\boldsymbol{h}}, \Sigma_{h} \forall h$ and $\boldsymbol{\pi}$ are estimated using MCMC. The remaining terms $\kappa_{h}, \Psi_{h}^{0}$, and $\nu_{h} \forall h$ and $\boldsymbol{\alpha}$ are hyperparameters.

This sort of representation incorporates typical prior distributions and a constrained likelihood model, and has been exploited frequently ${ }^{49,50,51}$ for its desirable posterior structure which is suitable for Gibbs sampling. Similarly here, by applying the necessary parameter constraints, defined by the latent association vectors, into the data model (Equation 8a), the parameters $\left(\boldsymbol{\mu}_{h}, \Sigma_{h}\right)$ possess the correct constraints in the posterior. That is, $\boldsymbol{\mu}_{h}$ follows a multivariate truncated normal 
distribution with truncation points dictated by the constraints defined in (8a), while $\Sigma_{h}$ follows the constrained inverse-Wishart distribution defined presently.

Let $\Sigma$ be distributed according to a $D$-dimensional constrained inverse-Wishart $\mathcal{I W}_{D}^{c}$ with constraints imposed by latent class $h$, and let $\mathcal{I} \mathcal{W}_{D}$ be an unconstrained $D$-dimensional inverseWishart density. Then

$$
\begin{aligned}
& f(\Sigma ; \Psi, \nu, h)=\mathcal{I}_{\mathcal{W}_{D}^{c}}^{c}(\Sigma ; \Psi, \nu, h) \\
& =C_{\mathcal{I} \mathcal{W}} \cdot \mathcal{I W}_{D}(\Sigma ; \Psi, \nu) \times \prod_{r<t} \mathbb{1}\left[\operatorname{sgn}\left(\Sigma_{r t}\right)=h_{[r]} \cdot h_{[t]}\right]
\end{aligned}
$$

where $C_{\mathcal{I} \mathcal{W}}$ is a normalizing constant.

We do inference on this model using a Metropolis Hastings within Gibbs algorithm, the details of which are in Supplementary File 1. With this procedure, we estimate $\boldsymbol{\pi}$ and $\boldsymbol{\mu}_{h}$ and $\Sigma_{h} \forall h$. Output from the pairwise fits are used to calculate hyperparameters $\boldsymbol{\alpha}, \boldsymbol{\mu}_{h}^{0}$, and $\Psi_{h}^{0}$ : computation of $\boldsymbol{\alpha}$ was described in Equation 6, while $\boldsymbol{\mu}_{h}^{0}$, and $\Psi_{h}^{0}$ are aggregations of pairwise parameter estimates constructed using a tactic described in $M C M C$ details. Parameters $\kappa_{h}$ and $\nu_{h} \approx n \alpha_{h}$, where $\alpha_{h}$ is the prior mixing weight for class $h$. We retain classes that satisfy $n \alpha_{h}>D$, since other such classes are unlikely to have members, and an inverse-Wishart distribution is only non-singular when the degrees of freedom are large enough.

\section{Testing consistency of effects}

The model fit output from CLIMB can be used to conduct hypothesis tests; in particular, we are interested in identifying consistency of signals across conditions. We propose a new test that generalizes the partial conjunction hypothesis test ${ }^{52}$, a standard hypothesis used for testing consistency, defined as

$$
\begin{aligned}
& \mathcal{H}_{0}^{u / D}:=\text { less than } u \text { out of } D \text { instances of the observed effect are non-null, versus } \\
& \mathcal{H}_{1}^{u / D}:=\text { at least } u \text { out of } D \text { instances of the observed effect are non-null }
\end{aligned}
$$

When seeking consistent signals, one may care not only about the significance of the signals, but also the sign of the effect. That is, if an observation is significantly positive in one experiment but significantly negative in another, then the observation should not be considered as consistent. Therefore, we propose a simple statistic for assessing the consistency of the sign of the effect across dimensions that generalizes the partial conjunction hypothesis to consider sign:

$$
\begin{array}{r}
\mathcal{H}_{0}^{u / D}:=\text { less than } u \text { out of } D \text { instances of the observed effect are concordant } \\
\text { with a specified association pattern, versus } \\
\mathcal{H}_{1}^{u / D}:=\text { at least } u \text { out of } D \text { instances of the observed effect are concordant } \\
\text { with a specified association pattern }
\end{array}
$$

To describe the rejection region $(R R)$ for this hypothesis, first define $h_{[d]}^{m}$ as the $d^{\text {th }}$ element of latent association vector $h_{m}$. Then, 


$$
\begin{aligned}
P^{u / D+} & :=\sum_{m=1}^{M} \operatorname{Pr}\left(\mathbf{x}_{i} \in h_{m} \mid \mathbf{x}\right) \cdot \mathbb{1}\left[\sum_{d=1}^{D} \mathbb{1}\left(h_{[d]}^{m}=1\right) \geq u\right] \\
P^{u / D 0} & :=\sum_{m=1}^{M} \operatorname{Pr}\left(\mathbf{x}_{i} \in h_{m} \mid \mathbf{x}\right) \cdot \mathbb{1}\left[\sum_{d=1}^{D} \mathbb{1}\left(h_{[d]}^{m}=0\right) \geq u\right] \\
P^{u / D-} & :=\sum_{m=1}^{M} \operatorname{Pr}\left(\mathbf{x}_{i} \in h_{m} \mid \mathbf{x}\right) \cdot \mathbb{1}\left[\sum_{d=1}^{D} \mathbb{1}\left(h_{[d]}^{m}=-1\right) \geq u\right]
\end{aligned}
$$

where $\operatorname{Pr}\left(\mathbf{x}_{i} \in h_{m} \mid \mathbf{x}\right)$ is the posterior probability of belonging to the class described by association vector $h_{m}$. We define $P^{u / D}=\max \left\{P^{u / D+}, P^{u / D 0}, P^{u / D-}\right\}$, and $R R:=\left\{\mathbf{x}: P^{u / D}>b\right\}$. For each observation, this calculation sums over its posterior probabilities of belonging to classes with association vectors indicating sufficient consistency.

Letting $T$ be the number of MCMC iterations retained after burn-in, the quantities in (12) are estimated as

$$
\begin{aligned}
& \hat{P}_{i}^{u / D+}=\frac{1}{T} \sum_{t=1}^{T}\left\{\sum_{m=1}^{M} \mathbb{1}\left(H_{i}^{(t)}=h_{m}\right) \cdot \mathbb{1}\left[\sum_{d=1}^{D} \mathbb{1}\left(h_{[d]}^{m}=1\right) \geq u\right]\right\} \\
& \hat{P}_{i}^{u / D 0}=\frac{1}{T} \sum_{t=1}^{T}\left\{\sum_{m=1}^{M} \mathbb{1}\left(H_{i}^{(t)}=h_{m}\right) \cdot \mathbb{1}\left[\sum_{d=1}^{D} \mathbb{1}\left(h_{[d]}^{m} \neq 0\right) \geq u\right]\right\} \\
& \hat{P}_{i}^{u / D-}=\frac{1}{T} \sum_{t=1}^{T}\left\{\sum_{m=1}^{M} \mathbb{1}\left(H_{i}^{(t)}=h_{m}\right) \cdot \mathbb{1}\left[\sum_{d=1}^{D} \mathbb{1}\left(h_{[d]}^{m}=-1\right) \geq u\right]\right\}
\end{aligned}
$$

for each observation $i$, leading to $\hat{P}_{i}^{u / D}=\max \left\{\hat{P}_{i}^{u / D+}, \hat{P}_{i}^{u / D 0}, \hat{P}_{i}^{u / D-}\right\}$, and we reject those $\mathbf{x}_{i}$ with $\hat{P}_{i}^{u / D}>b$. Large values of $\hat{P}_{i}^{u / D}$ correspond to consistent effects.

This test is flexible, and can be adapted to several purposes. For example, to test the typical partial conjunction hypothesis, one could modify the quantities in Equation 13 to

$$
\hat{P}_{i}^{u / D}:=\frac{1}{T} \sum_{t=1}^{T}\left\{\sum_{m=1}^{M} \mathbb{1}\left(H_{i}^{(t)}=h_{m}\right) \cdot \mathbb{1}\left[\sum_{d=1}^{D} \mathbb{1}\left(h_{[d]}^{m} \neq 0\right) \geq u\right]\right\} .
$$

In the analysis of VISION RNA-seq data, we tested for consistency in all -1, 0, and 1 groups. Thus, we applied our statistical test using all quantities in Equation 12 and letting $u=5$, such that $P^{5 / 5}=\max \left\{P^{5 / 5+}, P^{5 / 50}, P^{5 / 5-}\right\}$. Then, a differentially expressed gene is one that falls within the $R R:=\left\{\mathbf{x}: P^{5 / 5}<0.5\right\}$.

\section{Comparison against SCREEN and mash}

We used simulations to compare CLIMB to SCREEN ${ }^{16}$ and mash ${ }^{14}$, two methods designed for a similar purpose to CLIMB. SCREEN was designed specifically to test for consistent signals across many experiments. Like CLIMB, SCREEN employs a mixture model with classes governed by latent association vectors. SCREEN tackles the issue of computational intractability associated with these classes in two ways. First, it assumes the association vectors to be binary, rather than 
ternary. This reduces the growth rate of candidate latent classes to $2^{D}$, but comes at the cost of eliminating the method's ability to detect inverse associations and signs of effects. Second, SCREEN partitions the data's original conditions into clusters using a network community detection algorithm as an initial step, fitting separate models to each cluster. SCREEN next uses a heuristic to test for consistent signals across all conditions.

Mash, on the other hand, captures the relationship between observations across conditions through the covariances of each cluster in the mixture. Mash assumes the data come from a multivariate normal mixture, restricting each cluster to have zero mean. It sidesteps computational issues by not explicitly specifying the latent association vectors; instead, it models different clusters by specifying a list of candidate covariances which are generated a priori. Since the assumed distribution is symmetric and unimodal, model fitting is simplified to a convex optimization problem that can be computed efficiently.

In order to simulate data that mimic empirical data, we first fit CLIMB to the ChIP-seq and differential analysis of RNA-seq data described in VISION CTCF ChIP-seq and Shukla et al. ${ }^{53}$, respectively. Parameter estimates similar to those obtained from these model fits were used to simulate $n=15,000$ observations with 18 and 11 dimensions, respectively, according to the constrained normal mixture model in Equation 2 (see Supplementary Tables S4 - S8 and Supplementary Figures S20 and S21 for specific parameter settings for both simulations). Like the real datasets, these simulated data contain shared effects that are positively or negatively correlated across dimensions and effects that are unique to one dimension. We applied CLIMB, SCREEN, and mash to both datasets.

Though a usual goal of analyzing these types of data is to uncover the true association patterns of observations across conditions, of all methods, only CLIMB can report the full latent association vectors. To provide a fair comparison among methods, we test the partial conjunction hypothesis across a series of levels $u$. We do this as SCREEN's sole functionality is to test this hypothesis, while CLIMB and mash can be utilized for this purpose. By evaluating a range of $u$, we can obtain a comprehensive assessment of each method's ability to identify consistent signals at different levels of condition-specificity.

We assessed the performance of each method by comparing the identified consistent signals with the truth and computing the precision and recall at these thresholds (Supplementary Figures S2 and S3). Precision and recall were computed as

$$
\begin{gathered}
\text { precision }=\frac{\mid \text { significant effects } \cap \text { true effects } \cap \text { correctly signed } \mid}{\mid \text { significant effects } \mid} \\
\text { recall }=\frac{\mid \text { significant effects } \cap \text { true effects } \cap \text { correctly signed } \mid}{\mid \text { true effects } \mid}
\end{gathered}
$$

where significant effects are observations that have been estimated to be consistent, true effects are observations that truly are consistent, and correctly signed effects are observations whose true and estimated associations have the same sign. This computation is designed such that an effect correctly identified by an algorithm as significant, but whose effect was missigned, is considered a false positive. Analagous precision-recall curves that do not incorporate sign information are in Supplementary Figures S4 and S5. 


\section{Availability}

CLIMB is implemented in an $\mathrm{R}$ package, freely available on GitHub under an Artistic-2.0 license (https://github.com/hillarykoch/CLIMB). Examined datasets are available at NCBI's Gene Expression Omnibus (https://www.ncbi.nlm.nih.gov/geo/) ${ }^{54}$ under accession code GSE156074.

\section{Acknowledgments}

The authors are grateful for the support from their funding agencies: NIGMS training grant T32GM102057 (CBIOS training program to The Pennsylvania State University, to H.K.); NHGRI pre-doctoral fellowship 1F31HG010574 (to H.K.); NIGMS grant R01GM109453 (to Q.L.); NIDDK grant R24DK106766 (to R.C.H.). The authors thank Marit Vermunt and Gerd Blobel for generating and sharing the G1E CTCF ChIP-seq data used in this work.

\section{References}

1. Dimas, A. S. et al. Common regulatory variation impacts gene expression in a cell typedependent manner. Science 325, 1246-1250 (2009).

2. GTEx Consortium. The genotype-tissue expression (GTEx) pilot analysis: multitissue gene regulation in humans. Science 348, 648-660 (2015).

3. Morikawa, M. et al. ChIP-seq reveals cell type-specific binding patterns of BMP-specific Smads and a novel binding motif. Nucleic Acids Res 39, 8712-8727 (2011).

4. Arvey, A., Agius, P., Noble, W. S. \& Leslie, C. Sequence and chromatin determinants of cell-type-specific transcription factor binding. Genome Res 22, 1723-1734 (2012).

5. Wang, H. et al. Widespread plasticity in CTCF occupancy linked to DNA methylation. Genome Res 22, 1680-1688 (2012).

6. Neale, B. M. et al. Meta-analysis of genome-wide association studies of attentiondeficit/hyperactivity disorder. J Am Acad Child Psy 49, 884-897 (2010).

7. Yang, J. et al. Conditional and joint multiple-SNP analysis of GWAS summary statistics identifies additional variants influencing complex traits. Nat Genet 44, 369 (2012).

8. Voight, B. F. et al. Twelve type 2 diabetes susceptibility loci identified through large-scale association analysis. Nat Genet 42, 579 (2010).

9. Pharoah, P. D. et al. GWAS meta-analysis and replication identifies three new susceptibility loci for ovarian cancer. Nat Genet 45, 362 (2013).

10. Andreassen, O. A. et al. Improved detection of common variants associated with schizophrenia by leveraging pleiotropy with cardiovascular-disease risk factors. Am J Hum Genet 92, 197-209 (2013). 
11. Ernst, J., Nau, G. J. \& Bar-Joseph, Z. Clustering short time series gene expression data. Bioinformatics 21, i159-i168 (2005).

12. Gerrits, A. et al. Expression quantitative trait loci are highly sensitive to cellular differentiation state. PLoS Genet 5, e1000692 (2009).

13. Fu, J. et al. Unraveling the regulatory mechanisms underlying tissue-dependent genetic variation of gene expression. PLoS Genet 8, e1002431 (2012).

14. Urbut, S. M., Wang, G., Carbonetto, P. \& Stephens, M. Flexible statistical methods for estimating and testing effects in genomic studies with multiple conditions. Nature Genet 51, 187-195 (2019).

15. Heller, R., Yekutieli, D. et al. Replicability analysis for genome-wide association studies. Ann Appl Stat 8, 481-498 (2014).

16. Amar, D., Shamir, R. \& Yekutieli, D. Extracting replicable associations across multiple studies: Empirical Bayes algorithms for controlling the false discovery rate. PLoS Comput Biol 13, e1005700 (2017).

17. Flutre, T., Wen, X., Pritchard, J. \& Stephens, M. A statistical framework for joint eQTL analysis in multiple tissues. PLoS genetics 9, e1003486 (2013).

18. Wen, X. \& Stephens, M. Bayesian methods for genetic association analysis with heterogeneous subgroups: from meta-analyses to gene-environment interactions. Ann Appl Stat 8, 176 (2014).

19. Huang, T., Peng, H. \& Zhang, K. Model selection for Gaussian mixture models. Stat Sinica 27, 147-169 (2017).

20. Ferguson, J. P., Cho, J. H. \& Zhao, H. A new approach for the joint analysis of multiple ChIP-seq libraries with application to histone modification. Stat Appl Genet Mol 11 (2012).

21. Oudelaar, A. M. et al. Between form and function: the complexity of genome folding. Hum Mol Genet 26, R208-R215 (2017).

22. Philipsen, S. \& Hardison, R. C. Evolution of hemoglobin loci and their regulatory elements. Blood Cell Mol Dis 70, 2-12 (2018).

23. Xiang, G. et al. An integrative view of the regulatory and transcriptional landscapes in mouse hematopoiesis. Genome Res 30, 472-484 (2020).

24. Keller, C. A. et al. Effects of sheared chromatin length on ChIP-seq quality and sensitivity. bioRxiv preprint doi:10.1101/2020.09.30.320697 (2020).

25. Kim, T. H. et al. Analysis of the vertebrate insulator protein CTCF-binding sites in the human genome. Cell 128, 1231-1245 (2007).

26. Splinter, E. et al. CTCF mediates long-range chromatin looping and local histone modification in the $\beta$-globin locus. Gene Dev 20, 2349-2354 (2006). 
27. Behera, V. et al. Exploiting genetic variation to uncover rules of transcription factor binding and chromatin accessibility. Nature Commun 9, 1-15 (2018).

28. Essien, K. et al. CTCF binding site classes exhibit distinct evolutionary, genomic, epigenomic and transcriptomic features. Genome Biol 10, R131 (2009).

29. Landt, S. G. et al. ChIP-seq guidelines and practices of the ENCODE and modENCODE consortia. Genome Res 22, 1813-1831 (2012).

30. Plasschaert, R. N. et al. CTCF binding site sequence differences are associated with unique regulatory and functional trends during embryonic stem cell differentiation. Nucleic Acids Res 42, 774-789 (2013).

31. Villar, D., Flicek, P. \& Odom, D. T. Dynamics, mechanisms, and functional implications of transcription factor binding evolution in metazoans. Nat Rev Genet 15, 221 (2014).

32. Van Dongen, S. \& Enright, A. J. Metric distances derived from cosine similarity and Pearson and Spearman correlations. arXiv preprint doi:1208.3145 (2012).

33. McLean, C. Y. et al. GREAT improves functional interpretation of cis-regulatory regions. Nat Biotechnol 28, 495 (2010).

34. Love, M. I., Huber, W. \& Anders, S. Moderated estimation of fold change and dispersion for RNA-seq data with DESeq2. Genome Biol 15, 550 (2014).

35. Thomas, P. D. et al. PANTHER: a library of protein families and subfamilies indexed by function. Genome Res 13, 2129-2141 (2003).

36. Thomas, P. D. et al. Applications for protein sequence-function evolution data: mRNA/protein expression analysis and coding SNP scoring tools. Nucleic Acids Res 34, W645-W650 (2006).

37. Lindsay, B. G. Composite likelihood methods. Contem Math 80, 221-239 (1988).

38. Varin, C., Reid, N. \& Firth, D. An overview of composite likelihood methods. Stat Sinica 5-42 (2011).

39. Larribe, F. \& Fearnhead, P. On composite likelihoods in statistical genetics. Stat Sinica 43-69 (2011).

40. Cox, D. R. \& Reid, N. A note on pseudolikelihood constructed from marginal densities. Biometrika 91, 729-737 (2004).

41. Geys, H., Molenberghs, G. \& Ryan, L. M. Pseudolikelihood modeling of multivariate outcomes in developmental toxicology. J Am Stat Assoc 94, 734-745 (1999).

42. Fieuws, S., Verbeke, G., Boen, F. \& Delecluse, C. High dimensional multivariate mixed models for binary questionnaire data. J R Stat Soc C 55, 449-460 (2006).

43. Fieuws, S. \& Verbeke, G. Pairwise fitting of mixed models for the joint modeling of multivariate longitudinal profiles. Biometrics 62, 424-431 (2006). 
44. Molenberghs, G., Verbeke, G. \& Iddi, S. Pseudo-likelihood methodology for partitioned large and complex samples. Stat Probabil Lett 81, 892-901 (2011).

45. Vasdekis, V. G., Rizopoulos, D. \& Moustaki, I. Weighted pairwise likelihood estimation for a general class of random effects models. Biostatistics 15, 677-689 (2014).

46. de Bruijn, N. G. A combinatorial problem. Knaw Verhan 49, 758-764 (1946).

47. Good, I. J. Normal recurring decimals. J London Math Soc 1, 167-169 (1946).

48. Tarjan, R. Depth-first search and linear graph algorithms. SIAM J Comput 1, 146-160 (1972).

49. Wei, G. C. \& Tanner, M. A. Posterior computations for censored regression data. J Am Stat Assoc 85, 829-839 (1990).

50. Chib, S. Bayes inference in the Tobit censored regression model. J Econometrics 51, 79-99 (1992).

51. Albert, J. H. \& Chib, S. Bayesian analysis of binary and polychotomous response data. J Am Stat Assoc 88, 669-679 (1993).

52. Benjamini, Y. \& Heller, R. Screening for partial conjunction hypotheses. Biometrics 64, 1215-1222 (2008).

53. Shukla, S. A. et al. Comprehensive analysis of cancer-associated somatic mutations in class I HLA genes. Nat Biotechnol 33, 1152 (2015).

54. Barrett, T. et al. Ncbi geo: archive for functional genomics data sets-update. Nucleic acids research 41, D991-D995 (2012).

55. McLachlan, G. \& Peel, D. Finite mixture models (John Wiley \& Sons, New York, 2004).

56. Fan, J. \& Li, R. Variable selection via nonconcave penalized likelihood and its oracle properties. J Am Stat Assoc 96, 1348-1360 (2001).

57. Geweke, J. Efficient simulation from the multivariate normal and student-t distributions subject to linear constraints and the evaluation of constraint probabilities. In Comp Sci Stat, 571-578 (American Statistical Association, 1991).

58. Li, Y. \& Ghosh, S. K. Efficient sampling methods for truncated multivariate normal and student-t distributions subject to linear inequality constraints. J Stat Theory Pract 9, 712-732 (2015).

59. Wijsman, R. A. Random orthogonal transformations and their use in some classical distribution problems in multivariate analysis. Ann Math Stat 415-423 (1957).

60. Shaby, B. \& Wells, M. T. Exploring an adaptive Metropolis algorithm. Tech. Rep., Dept. Statistical Science, Duke Univ., Durham, NC. (2011). 
61. McLachlan, G. J., Bean, R. \& Jones, L. B.-T. A simple implementation of a normal mixture approach to differential gene expression in multiclass microarrays. Bioinformatics 22, 1608-1615 (2006).

62. Bovy, J., Hogg, D. W., Roweis, S. T. et al. Extreme deconvolution: Inferring complete distribution functions from noisy, heterogeneous and incomplete observations. Ann Appl Stat 5, 1657-1677 (2011).

63. Zhang, Y. et al. Model-based analysis of ChIP-Seq (MACS). Genome Biol 9, R137 (2008).

64. ENCODE Project Consortium. An integrated encyclopedia of DNA elements in the human genome. Nature 489, 57-74 (2012).

65. Quinlan, A. R. \& Hall, I. M. BEDTools: a flexible suite of utilities for comparing genomic features. Bioinformatics 26, 841-842 (2010).

66. Xiang, G. et al. S3norm: simultaneous normalization of sequencing depth and signal-to-noise ratio in epigenomic data. Nucleic Acids Res 48, e43-e43 (2020).

67. Li, B. \& Dewey, C. N. RSEM: accurate transcript quantification from RNA-Seq data with or without a reference genome. BMC Bioinformatics 12, 323 (2011). 